\title{
Influence of Urban Buildings Layout on Contaminant Dispersion of Heating Exhaust
}

\author{
Yongxin Liü ${ }^{1, a}$, Changhong Zhan ${ }^{1, b}$ and Hong $\operatorname{Jin}^{1, \mathrm{c}}$ \\ ${ }^{1}$ School of Architecture, Harbin Institute of Technology; Heilongjiang Cold Region Architectural \\ Science Key Laboratory, Harbin 150090, China. \\ aaces1982@sina.com, bzhan_changhong@163.com, chongjin777@sina.com
}

Keywords: Air pollution, heating exhaust, urban planning, severe cold zones.

\begin{abstract}
China has a vast territory of severe cold zones, occupying a quarter of the total land. Heating exhaust from coal heating boilers is an important influence factor of air quality in the winter. This situation will be relieved by some urban planning methods. There is no research about air pollution caused by heating exhaust. In this paper, four governing equations (Continuity equation, Momentum equation, Energy equation and Mass transport equation) are introduced to study contaminant dispersion of heating exhaust. Computational Fluid Dynamics (CFD) is used to compute outdoor wind environment and air pollution condition. In meshing process, a size function is applied to reduce number of grids and improve computational accuracy. Simulation result can achieve influence analysis of air pollution on key elements of urban planning. Study results can provide theory foundation for urban planning.
\end{abstract}

\section{Introduction}

China has a vast territory of severe cold zones, which occupies a quarter of the total land. With the expanding of urbanization, changes and deterioration of living environment has been the key issue of the urbanization development in severe cold zones.

The quality of the air quality seriously affects comfort feeling of the living environment. Air quality is relatively poor in severe cold zones. We choose a city Harbin located in the northeast of China as an example. In 2015, there are 42 heavy pollution days in this year, and heavy pollution centers in winter [1]. Y. L. Zhang [2] concluded that the concentrations of air contaminants including $\mathrm{SO}_{2}, \mathrm{NO}_{2}$ and a large number of particulate matters in the winter than summer in part of severe cold zones. This period of time coincides with heat-supply seasons. So, heating exhaust can be seen as an important influence factor of air quality in the winter.

In China, the reserves of coal can be accounted for $58.8 \%$ in the total reserves. Objectively, this energy consumption pattern depending on coal will exist for a long time [3]. At present, in China heating boilers are still mainly coal based. Taking into account the economic level and coal reserves, this situation can not be improved in the near future. The adverse effects of coal-fired to environment will exist for a long time. In severe cold zones of China, outdoor temperature is lower than $0{ }^{\circ} \mathrm{C}$ during the winter. There are frozen water and dry plants except for a small part of trees, such as pine. These climatic conditions form unique characteristics of urban surface in this zone. These characteristics lower the absorption and adsorption of harmful gases and particulates. Contaminant dispersion under these conditions also has certain special characteristics.

In previous studies, of heating sources focuses on the dominant wind direction in winter. If the site selection is limited, there is no research on air quality and urban environment influenced by urban planning. To improve urban air quality during the heating season, it is necessary to reduce the negative consequences by reasonable buildings layout and heating planning on the urban planning stage.

There are some main research methods on urban physical environment optimization, such as computational fluid dynamics, remote sensing, field measurement and wind tunnel experiment. Their advantages and disadvantages are listed below. Computational fluid dynamics (CFD): High 
efficiency, low consumption and avoiding accidental factors of weather conditions; Comparison and verification are needed under complex environment conditions. Remote sensing: The analysis scope is big enough for urban planning study, and with High accuracy and reliability; Low consumption and may be affected by meteorological conditions. Field measurement: data obtained directly are more reliability; and may be affected by causal factors. Wind tunnel experiment: data with high accuracy, boundary condition is easy to control; also needs large investment of manpower and material resources. When multi-physical field needs to be considered, application of the method is limited.

In this paper, the CFD simulation technology is used to study contaminant diffusion model and dispersion of heating exhaust in severe cold area of China. Then, the influence of several typical architectural layouts is compared, which provides the theoretical support and guidance for urban and heating planning.

\section{Numerical Model}

\subsection{Governing equations}

The CFD simulations were performed through the commercial software Ansys Fluent 15.0 using: 3D double precision solver, pressure based solver, steady-state analysis and RANS steady equations were solved in combination with the standard $k-\varepsilon$ model. The DPM model was used for contaminant dispersion. These are the equations governing the phenomenon.

This leads us to the continuity equation for incompressible flows:

$$
\frac{\partial \bar{u}_{i}}{\partial x_{i}}=0
$$

The time-averaged Navier-Stokes equations for incompressible flows are

$$
\bar{u}_{j} \frac{\partial \bar{u}_{i}}{\partial x_{j}}=-\frac{1}{\rho} \frac{\partial \bar{p}}{\partial x_{i}}+\frac{\mu}{\rho} \frac{\partial^{2} \bar{u}_{i}}{\partial x_{i} \partial x_{j}}-\frac{\partial}{\partial x_{j}}\left(\bar{u}_{i} \bar{u}_{j}^{\prime}\right)+f_{i}
$$

Energy Equation can be expressed as

$$
\bar{u}_{i} \frac{\partial \bar{T}}{\partial x_{i}}+\frac{\partial}{\partial x_{i}}\left(K_{T} \frac{\partial \bar{T}}{\partial x_{i}}\right)=0
$$

where $\bar{u}_{i}$ is the mean velocity of the air flow; $\bar{u}_{i} \bar{u}_{j}^{\prime}$ is the Reynolds stress tensor, $\rho$ the air density, $\bar{p}$ is the mean pressure, $\mu$ molecular viscosity, $f_{i}$ the thermal-induced buoyant force, $\bar{T}$ potential temperature and $K_{T}$ is the heat diffusivity. The $k-\varepsilon$ model was chosen as turbulence model, expressing the Reynolds stress tensor $\bar{u}_{i} \bar{u}_{j}^{\prime}$. The turbulent kinetic energy, $k$, and its rate of dissipation, $\varepsilon$, were obtained through the following transport equations:

$$
\begin{aligned}
& \frac{\partial}{\partial x_{i}}\left(k \bar{u}_{i}\right)=\frac{1}{\rho} \frac{\partial}{\partial x_{j}}\left(\left(\mu+\frac{\mu_{t}}{\sigma_{k}}\right) \frac{\partial k}{\partial x_{j}}\right)+\frac{1}{\rho} G_{k}+\frac{1}{\rho} G_{b}-\varepsilon \\
& \frac{\partial}{\partial x_{i}}\left(\varepsilon \bar{u}_{i}\right)=\frac{1}{\rho} \frac{\partial}{\partial x_{j}}\left(\left(\mu+\frac{\mu_{t}}{\sigma_{\varepsilon}}\right) \frac{\partial \varepsilon}{\partial x_{j}}\right)+\frac{1}{\rho} C_{1 \varepsilon} \frac{\varepsilon}{k}+\frac{1}{\rho}\left(G_{k}+C_{3 \varepsilon} G_{b}\right)-G_{2 \varepsilon} \frac{\varepsilon^{2}}{k}
\end{aligned}
$$

where $G_{k}$ is the turbulent kinetic energy caused by the average velocity gradients; $G_{b}$ is the turbulent kinetic energy caused by buoyancy; $C_{1 \varepsilon}, C_{2 \varepsilon}$ and $C_{3 \varepsilon}$ are constants and the expressions of $K_{T}$ and $\mu_{t}$ are reported in the Ansys Fluent $15.0 k-\varepsilon$ model [4]; $\sigma_{k}$ and $\sigma_{\varepsilon}$ are the turbulent Prandtl numbers respectively for $k$ and $\varepsilon$.

In the calculation of incompressible flows, the energy equation is decoupled from the continuity equation and the Navier-Stokes equations; i.e. equations (1) to (2) may be solved first, and then used 
in the energy equation, together with the knowledge of $\bar{u}_{i}$, and $\bar{p}$, to determine the temperature field [5].

For quantification research of contaminant dispersion, Mass transport equation is introduced in this paper.

$$
\bar{u}_{i} \frac{\partial \bar{c}}{\partial x_{i}}+\frac{\partial}{\partial x_{i}}\left(D \frac{\partial \bar{c}}{\partial x_{i}}\right)=0
$$

where $\bar{c}$ is the mean concentration of the contaminant.

\subsection{Discrete Phase Model (DPM)}

Contaminant dispersion of single heating exhaust is a three-dimensional, non phase transformation, incompressible, steady, and multi-component turbulent flow, heat and mass transfer process. The fluid phase is treated as a continuum by solving the Navier-Stokes equations, while the dispersed phase is solved by tracking a large number of particles through the calculated flow field. The dispersed phase can exchange momentum, mass, and energy with the fluid phase. In details, continuity equation, momentum equation (three), energy equation and mass transport equation need to be considered.

In addition to solving transport equations for the continuous phase, ANSYS Fluent allows users to simulate a discrete second phase in a Lagrangian frame of reference. We choose DPM to solve our subjects. Calculation of the discrete phase trajectory using a Lagrangian formulation that includes the discrete phase inertia, hydrodynamic drag, and the force of gravity for steady flow. The initial conditions for the discrete phase calculations in ANSYS Fluent need to be input, such as the starting positions, velocities, particle properties, and other parameters for a particle stream. Since the volume rate of particles in heating exhaust is less than $10 \%$, uncoupled calculation is chosen as the tool of contaminant dispersion of heating exhaust. Uncoupled calculation is adequate when the discrete phase is present at a low mass and momentum loading, in which case the continuous phase is not impacted by the presence of the discrete phase.

For the uncoupled calculation, we will perform the following two steps:

(1) Solve the continuous phase flow field using Eq. (1), (2) and (3).

(2) Calculate and plot the particle trajectories for discrete phase injections of interest using Eq. (6), based on a fixed continuous-phase flow field.

\section{Calculation Model}

\subsection{Regional Climate}

Harbin has a cold climate with a winter mean temperature of $-18.4{ }^{\circ} \mathrm{C}(254.75 \mathrm{~K})$, and the atmospheric pressure is $99.36 \mathrm{KPa}$ in the winter. The prevailing wind direction is SSW in the winter. The average wind speed is $3.2 \mathrm{~m} / \mathrm{s}$. The wind collecting devices are usually installed at $10 \sim 12 \mathrm{~m}$ from the ground. The space wind speed can be written as follow.

$$
u_{z}=u_{0} \cdot\left(z / z_{0}\right)^{0.22} \quad\left(u_{0}=3.2 \mathrm{~m} / \mathrm{s} \text {, when } z_{0}=10 \mathrm{~m}\right)
$$

Combing field research and industry standards, the situation of heating exhaust can be concluded as follow. The height of chimney is $100 \mathrm{~m}$, and the inner diameter is $2 \mathrm{~m}$. The temperature of heating exhaust is $100^{\circ} \mathrm{C}(373.15 \mathrm{~K})$, and the velocity is $15 \mathrm{~m} / \mathrm{s}$. The density, specific heat capacity, thermal conductivity and viscosity of heating exhaust are $\rho=0.88 \mathrm{~kg} / \mathrm{m}^{3}, C_{p}=1.079 \mathrm{~kJ} /\left(\mathrm{kg} .{ }^{\circ} \mathrm{C}\right), \lambda$ $=3.508 \times 10^{-2} \mathrm{~W} /\left(\mathrm{m}^{\circ} \mathrm{C}\right)$ and $\mu=22 \times 10^{-6} \mathrm{~kg} /(\mathrm{ms})$ respectively. Physical parameters of particulate matters included in heating exhaust are $\rho=2000 \mathrm{~kg} / \mathrm{m}^{3}, C_{p}=0.907 \mathrm{~kJ} /\left(\mathrm{kg} .{ }^{\circ} \mathrm{C}\right)$ and $d=2 \times 10^{-6} \mathrm{~m}$. The mass flow of particulate matters is $0.487 \mathrm{~kg} / \mathrm{s}$.

\subsection{Mesh and Boundary Conditions}

The simulation domain was set as $2000 \mathrm{~m}(x) \times 1000 \mathrm{~m}(y) \times 600 \mathrm{~m}(z)$ due to the limited computer capacity and the mesh size around buildings was set as $1 \mathrm{~m}$. 
The total number of cells was $5.43 \times 10^{5}$ for the coarse, $1.83 \times 10^{6}$ for the basic and $4.86 \times 10^{6}$ for the fine grid. For uniform reporting of the grid-convergence study and for an estimation of the grid-induced discretization error, the Grid Convergence Index (GCI) was tested. There is no significant difference on simulation results, so the basic grid was chosen herein. Size function was introduced in our study, so the minimum grid on the outlet of the chimney can reach $0.05 \mathrm{~m}$. In the simulation domain, the maximum grids are 20m, locating in the outer of the domain. Fig. 1 shows horizontal distribution of grid. A PC with 8GB RAM and an Intel i-5 processor were used for the simulation.

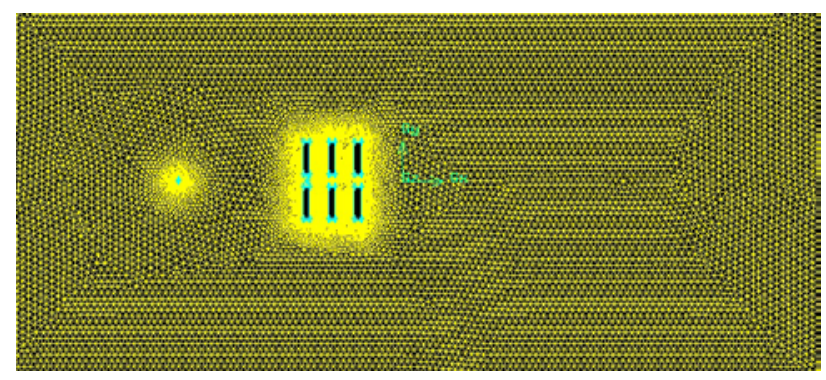

Fig. 1 Grid on $1.5 \mathrm{~m}$ height

Flow boundary conditions are listed as follow. The left surface is set velocity inlet; the right surface is set pressure outlet; side and upper surfaces are set symmetry; surface of the chimney outlet is set velocity inlet; the other surfaces are set wall. In calculation, the residuals of all parameters are set to $10^{-4}$. The initial positions of the particulate matters will be the location of the data points on the specified surface (the chimney outlet). Type surface injections were chosen in Fluent 15.0. Grids were generated by Gambit (r 2.4.6). Those two softwares are used in CFD simulation of heating exhaust contaminant dispersion.

\section{Simulation Analysis}

In this paper, we mainly consider contaminant dispersion in the residential area, composing of multi-story buildings. Each building size is $10 \mathrm{~m}(x) \times 50 \mathrm{~m}(y) \times 30 \mathrm{~m}(\mathrm{z})$. In the regional planning, the main influence factors are listed as follow. (1) Location of heating sources: determine the distance between the chimney and the building group. (2) Distance between buildings and the form of enclosure: affect the process of contaminant dispersion. This paper focuses on the air pollution situation of the main wind down wind direction in winter, especially considering the wind speed and the pollution on the pedestrian height (above ground $1.5 \mathrm{~m}$ ).

\subsection{Location of Heating Exhaust}

Considering geometrical dimensions of the chimney and different relative positions of buildings studied, we set the chimney 2 times, 4 times and 6 times of building heights upwind away from building. Then, local wind speeds and contaminant dispersion are compared respectively. The results of numerical simulation are listed as follows.

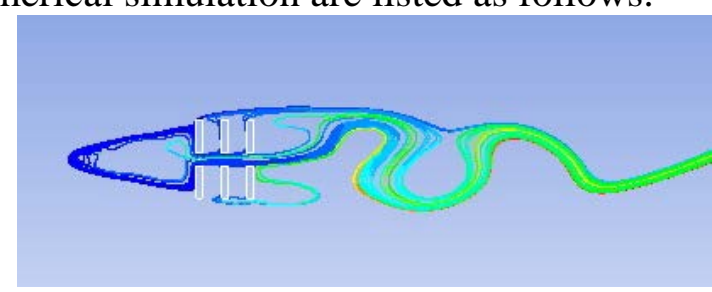

(a) Particle trace when the distance is $200 \mathrm{~m}$

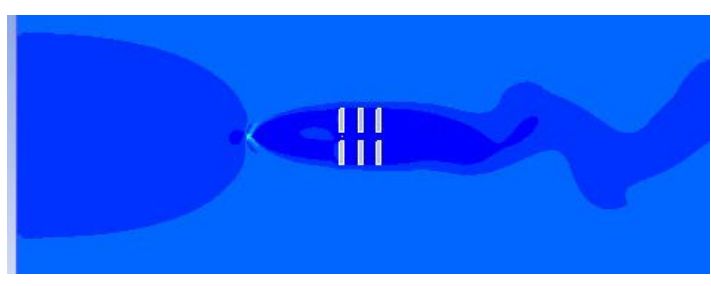

(b) Velocity vector when the distance is $200 \mathrm{~m}$ 


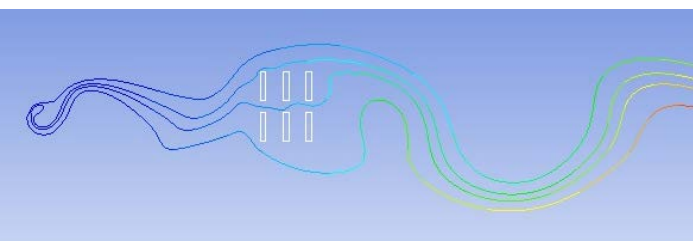

(c) Particle trace when the distance is $400 \mathrm{~m}$

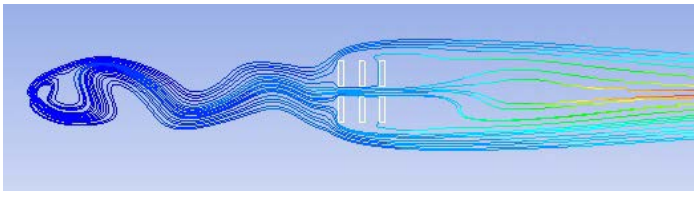

(e) Particle trace when the distance is $600 \mathrm{~m}$

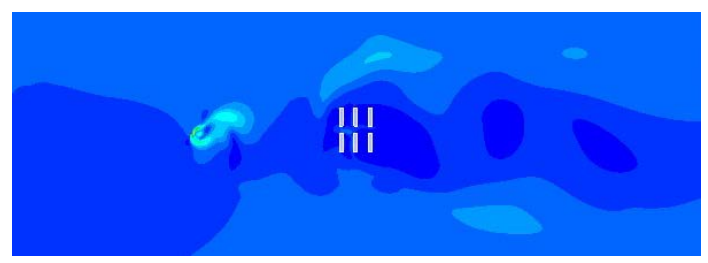

(d) Velocity vector when the distance is $400 \mathrm{~m}$

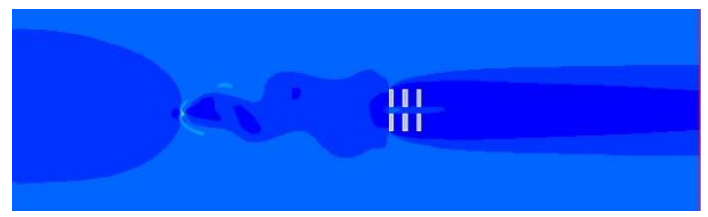

(f) Velocity vector when the distance is $600 \mathrm{~m}$

Fig. 2. Contaminant dispersion under different distances between heating exhaust and buildings

As shown in results, when the distance between a heating exhaust and a building is $400 \mathrm{~m}$, being 4 times the height of the chimney, the condition of wind environment around the building is better. Moreover, dispersion condition of particle pollutants is more favorable. Thus, the air quality is better. Increasing between a heating exhaust and a building further will not improve the air quality. Next, other study results will be given under this distance.

\subsection{Space between Buildings}

Sunshine is important for northern residents. In those areas, buildings prefer south to north direction. Area layout contains buildings in lines or rows. Different spaces (distances) between buildings (30m, 45m, $60 \mathrm{~m})$, the ratio of building space and building height $(1: 1,1.5: 1,2: 1)$ will affect the local air quality. When the distance between buildings is $30 \mathrm{~m}$, contaminant dispersion is shown in Figure 2 (c) and (d). Other two numerical simulation results are listed as follows.

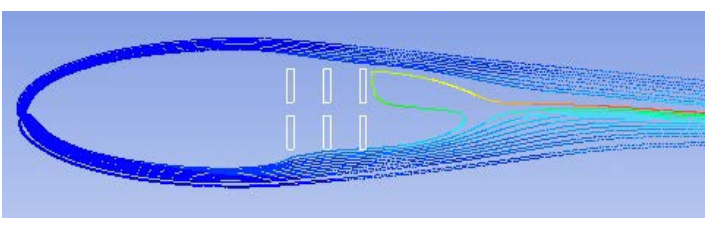

(a) Particle trace when the distance is $45 \mathrm{~m}$

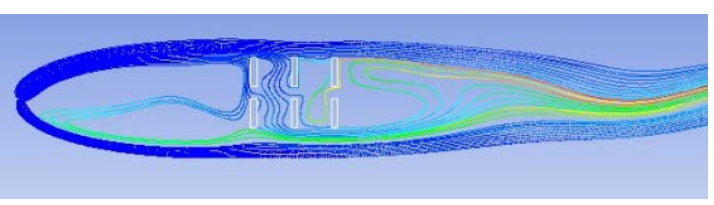

(c) Particle trace when the distance is $60 \mathrm{~m}$

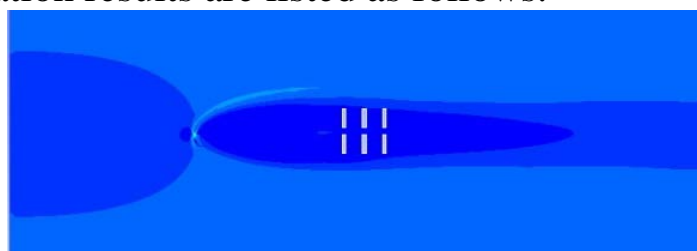

(b) Velocity vector when the distance is $45 \mathrm{~m}$

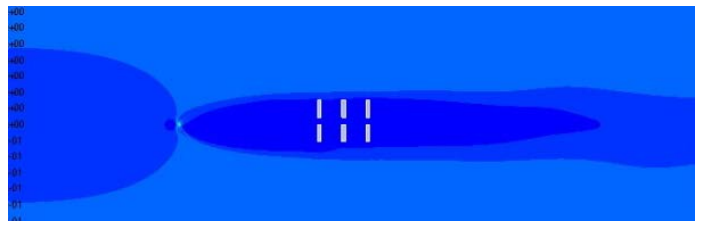

(d) Velocity vector when the distance is $60 \mathrm{~m}$

Fig. 3. Effect of contaminant dispersion under different space between buildings

Since area layouts are almost same, there is no significant difference between velocity vectors. But Particle traces are different from each other. Comparing Figure 2 (c), Figure 3 (a) and Figure 3 (c), we can be found that when the distance between two buildings is 1.5 times the height of buildings, fly ash pollutant distribution in this zone is less, and air quality is better.

\subsection{Buildings Area Layout}

We consider contaminant dispersion affected by three typical buildings area layouts, which are enclosed, staggered and determinant regions. The contaminant dispersion in the determinant layout is shown in Figure 3 (a) and (b). Other numerical simulation results are listed as follows. 


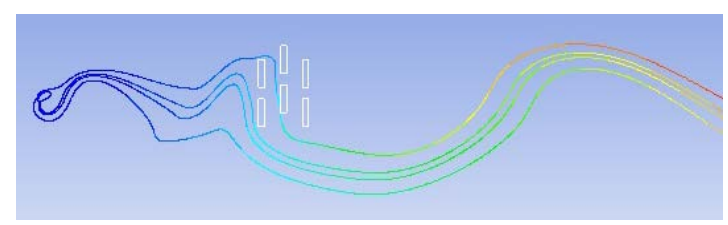

(a) Particle trace of staggered layout

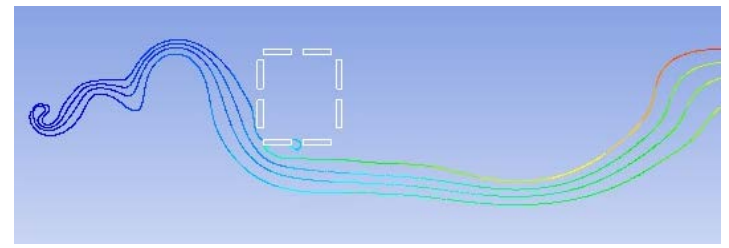

(c) Particle trace of enclosed layout

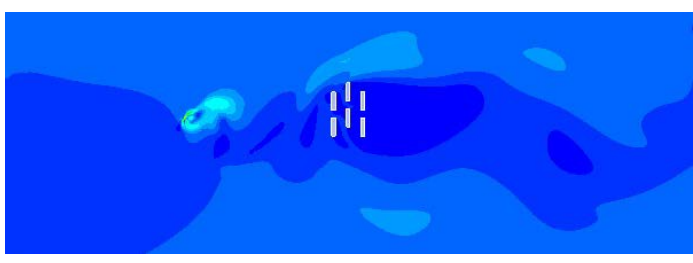

(b) Velocity vector of staggered layout

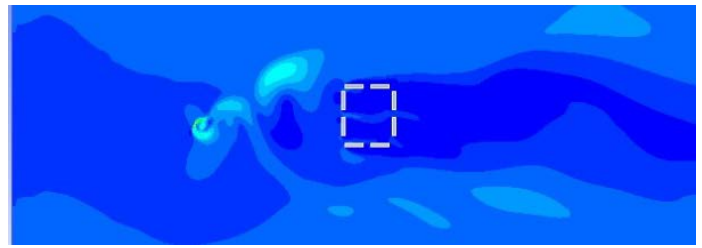

(d) Velocity vector of enclosed layout

Fig. 4. Affections of contaminant dispersion by different buildings area layout

According to velocity vectors, the determinant and staggered layouts are better. Then, according to particle trace, the determinant and enclosed layouts are better for improving air quality and reducing the adverse effect of exhaust pollutants in this zone. Considering the situation of outdoor wind environment and contaminant dispersion together, the determinant layout is more favorable for protection from heating exhaust in severe cold zones.

\section{Summary}

If the area size is big enough, there are three urban planning principles which are better for protection from heating exhaust and improving air quality. (1) Keep a reasonable distance between a chimney and buildings. (2) Arrange buildings as determinant layout. (3) Keep the space between buildings is 1.5 times of the height of buildings.

\section{Acknowledgements}

The research was supported by The Fundamental Research Funds for the Central Universities (Grant No. HIT. NSRIF. 2015073) and Heilongjiang Postdoctoral Financial assistance Fund LBH-Z15083.

Liu Yongxin (corresponding author), Ph. D., Postdoctor, Lecturer, Harbin Institute of Technology, aces1982@sina.com.

\section{References}

[1] Information on http://hlj.sina.com.cn/news/b/2016-04-22/detail-ifxrpvcy4320728.shtml.

[2] Y.L. Zhang, The research about Xi'an concentrated heating flue gas emission and diffusion, Chang'an University, 2013.

[3] Q. Xin, The completion of major power generation in the whole country, China Power Enterprise Management. 8 (2010) 95.

[4] Ansys Fluent Theory Guide 15.0, ANSYS, Inc., Southpointe 275 Technology Drive Canonsburg, PA, USA, 2013, pp. 1341-1348.

[5] S.S. Antman, J.E. Marsden and L. Sirovich. Applied Mathematical Sciences Volume 158, Prandtl-Essentials of Fluid Mechanics (Third Edition), Springer, 2010, pp. 277-278. 